Pacific Journal of Mathematics

THE BOUNDARY BEHAVIOR OF HENKIN'S KERNEL

CK ROBERT A HERN AND ROBERT Bruce SCHNeIDER 


\title{
THE BOUNDARY BEHAVIOR OF HENKIN'S KERNEL
}

\section{Patrick Ahern and Robert Schneider}

\begin{abstract}
In this paper, the boundary behaviour of a reproducing kernel, introduced by Henkin, for strictly pseudoconvex domains is studied. As an application, an improved version of a known result about generators of certain maximal ideals is given.
\end{abstract}

The boundary behaviour of the Bergmann kernel $B(z, \zeta)$ for a strictly pseudoconvex domain has been studied by Bergmann [1] and Hörmander [5]. Among other things, they determine the rate at which $B(z, z)$ goes to infinity as $z$ approaches a boundary point of the domain. Another type of reproducing kernel has been introduced by Henkin [3] for bounded strictly pseudoconvex domains $D$, in $\mathbf{C}^{n}$. Henkin's kernel is of the form $K(\zeta, z) / \Phi^{n}(\zeta, z)$, where $K$ and $\Phi$ are holomorphic in a neighborhood of $\bar{D}$ for each $\zeta$ in $\partial D$, the boundary of $D$. The denominator $\Phi$ has the properties that $\Phi(\zeta, \zeta)=0$ for all $\zeta \in \partial D$ and that $\Phi(\zeta, z) \neq 0$ if $z \in \bar{D} \backslash\{\zeta\}$. For $z$ near $\zeta$, $\Phi$ is given explicitly (up to a nonvanishing factor) in terms of the plurisubharmonic function $\rho$ that defines the domain $D$. Precise statements about the way $\Phi(\zeta, z)$ approaches zero as $z$ approaches $\zeta$ from inside $D$ are given in Henkin's paper [3]. We show that this determines the behaviour of the kernel $K / \Phi^{n}$ by showing that $K(\zeta, \zeta) \neq 0$.

It has been proven in [4], [6], [7] and [9], that if $f$ is in the space $A(D)$ of functions continuous on $\bar{D}$ and holomorphic in $D$ and if $a \in D$ then there exist functions $g_{1}, \cdots, g_{n} \in A(D)$ such that

$$
f(z)-f(a)=\sum_{j=1}^{n}\left(z_{\jmath}-a_{j}\right) g_{\jmath}(z)
$$

This is a solution to a problem originally posed by Gleason [2] for the unit ball in $\mathbf{C}^{n}$. Using Henkin's integral formula and our result on the behaviour of Henkin's kernel we can improve the result just stated in two ways. Firstly, we show that the $g_{\imath}$ can be chosen in such a way that the association between $f$ and the $n$-tuple of functions $\left(g_{1}, \cdots, g_{n}\right)$ is linear, and secondly we show that the $g_{\imath}$ may be also chosen to depend analytically on $a$ as well as on $z$.

1. Notation. $D$ will always denote a bounded strictly pseudoconvex domain in $\mathbf{C}^{n}$ defined as $D=\{z: \rho(z)<0\}$, where $\rho$ is defined and strictly plurisubharmonic in a neighborhood $U$ of $\bar{D}$, such that the gradient of $\rho$ is not zero on the boundary of $D$. For $\epsilon>0$ we let 
$D_{\epsilon}=\{z \in U: \rho(z)<\epsilon\}$ and if $V$ is a neighborhood of $\partial D$ we let $V_{\epsilon}=V \cap D_{\epsilon}$. We denote by $C^{k}\left(V_{\epsilon}, H\left(D_{\epsilon}\right)\right)$ the space of $C^{k}$ functions on $V_{\epsilon}$ with values in the space $H\left(D_{\epsilon}\right)$ of functions holomorphic in $D_{\epsilon}$. In other words, functions that are $C^{k}$ on $V_{\epsilon} \times D_{\epsilon}$ and holomorphic in $D_{\epsilon}$ for each fixed $\zeta \in V_{\epsilon}$. Finally, we let $S_{z, \delta}=\{\zeta:|\zeta-z|<\delta\}$.

2. The work of Henkin [3], modified slightly by $\emptyset v$ relid [8], shows that if $D$ has a $C^{3}$ boundary then there are functions $K$ and $\Phi$ and a neighborhood $V$ of $\partial D$ and an $\epsilon>0$ such that:

2.1. (a) $K \in C^{1}\left(V_{\epsilon}, H\left(D_{\epsilon}\right)\right)$ and

$$
\Phi \in C^{2}\left(V_{\epsilon}, H\left(D_{\epsilon}\right)\right) \text {. }
$$

(b) $\Phi(\zeta, z) \neq 0$ if $z \in \bar{D} \backslash\{\zeta\}$.

2.2. If $f \in A(D)$ then

$$
f(z)=\int_{\partial D} f(\zeta) \frac{K(\zeta, z)}{\Phi^{n}(\zeta, z)} d \sigma(\zeta), \quad \text { for all } z \in D
$$

where $d \sigma$ is $2 n-1$ dimensional volume measure on $\partial D$.

2.3. There are constants $\gamma, \delta_{0}>0$ such that for all $z \in \bar{D}$ and $0<\delta<\delta_{0}$,

$$
\int_{\partial D \cap S_{z, \delta}} \frac{|\zeta-z|}{\left|\Phi^{n}(\zeta, z)\right|} d \sigma(\zeta) \leqq \gamma \delta \log \frac{1}{\delta}
$$

TheOREM A. Suppose $K$ and $\Phi$ satisfy properties 2.1, 2.2, and 2.3, then $K\left(\zeta_{0}, \zeta_{0}\right) \neq 0$ for any $\zeta_{0} \in \partial D$.

Proof. We assume that $K\left(\zeta_{0}, \zeta_{0}\right)=0$ and arrive at a contradiction. If $K\left(\zeta_{0}, \zeta_{0}\right)$ were zero then, from property 2.1 , there would be a constant $M$ such that

(a) $\left|K\left(\zeta, \zeta_{0}\right)\right| \leqq M\left|\zeta-\zeta_{0}\right|$,

(b) $\left|K(\zeta, z)-K\left(\zeta_{0}, z\right)\right| \leqq M\left|\zeta-\zeta_{0}\right|$,

(c) $\left|K\left(\zeta_{0}, z\right)\right| \leqq M\left|z-\zeta_{0}\right|$.

Now it follows from (a) and 2.3 that

$$
\int_{\partial D} \frac{\left|K\left(\zeta, \zeta_{0}\right)\right|}{\left|\Phi^{n}\left(\zeta, \zeta_{0}\right)\right|} d \sigma(\zeta)<\infty
$$

We will show that if $f \in A(D)$, then 
2.4. $\quad f\left(\zeta_{0}\right)=\int_{\partial D} f(\zeta) \frac{K\left(\zeta, \zeta_{0}\right)}{\Phi^{n}\left(\zeta, \zeta_{0}\right)} d \sigma(\zeta)$

Due to the remark just made, the right hand side of 2.4 is welldefined. To prove 2.4 we show that as $z$ approaches $\zeta_{0}$ in a certain way, the expression,

$$
f(z)-\int_{\partial D} f(\zeta) \frac{K\left(\zeta, \zeta_{0}\right)}{\Phi^{n}\left(\zeta, \zeta_{0}\right)} d \sigma(\zeta)
$$

converges to 0 . Now by 2.2 we have,

$$
\begin{array}{rl}
f(z)-\int_{\partial D} & f(\zeta) \frac{K\left(\zeta, \zeta_{0}\right)}{\Phi^{n}\left(\zeta, \zeta_{0}\right)} d \sigma(\zeta)=\int_{\partial D} f(\zeta)\left[\frac{K\left(\zeta, \zeta_{0}\right)}{\Phi^{n}\left(\zeta, \zeta_{0}\right)}-\frac{K(\zeta, z)}{\Phi^{n}(\zeta, z)}\right] d \sigma(\zeta) \\
= & \int_{\partial D \backslash s_{\zeta_{0}, \delta}} f(\zeta)\left[\frac{K\left(\zeta, \zeta_{0}\right)}{\Phi^{n}\left(\zeta, \zeta_{0}\right)}-\frac{K(\zeta, z)}{\Phi^{n}(\zeta, z)}\right] d \sigma(\zeta) \\
& +\int_{\partial D \cap s_{\xi_{0}, \delta}} f(\zeta)\left[\frac{K\left(\zeta, \zeta_{0}\right)}{\Phi^{n}\left(\zeta, \zeta_{0}\right)}-\frac{K(\zeta, z)}{\Phi^{n}(\zeta, z)}\right] d \sigma(\zeta)
\end{array}
$$

Now for any fixed $\delta>0$, the first integral above approaches zero as $z$ approaches $\zeta_{0}$, since we can take the limit under the integral sign. As for the second integral, its absolute value is not greater than

$$
\int_{\partial D \cap s_{\varepsilon_{0}, \delta}}|f(\zeta)| \frac{\left|K\left(\zeta, \zeta_{0}\right)\right|}{\left|\Phi^{n}\left(\zeta, \zeta_{0}\right)\right|} d \sigma(\zeta)+\int_{\partial D \cap s_{\zeta_{0} . \delta}}|f(\zeta)| \frac{|K(\zeta, z)|}{\left|\Phi^{n}(\zeta, z)\right|} d \sigma(\zeta)
$$

Now by (a) and 2.3 , the first of these integrals is majorized by $M\|f\|_{\infty} \gamma \delta \log 1 / \delta$. To estimate the second of these integrals we let $z$ approach $\zeta_{0}$ along the inward normal to $\partial D$. Now if $z$ lies on this normal and if $\delta$ is sufficiently small then there is a constant $C$ such that $\left|z-\zeta_{0}\right| \leqq C|z-\zeta|$ and $\left|\zeta-\zeta_{0}\right| \leqq C|z-\zeta|$ as long as $\left|z-\zeta_{0}\right|<\delta$ and $\left|\zeta-\zeta_{0}\right|<\delta$, and hence $|K(\zeta, z)| \leqq\left|K(\zeta, z)-K\left(\zeta_{0}, z\right)\right|+\left|K\left(\zeta_{0}, z\right)\right| \leqq$ $M\left|\zeta-\zeta_{0}\right|+M\left|z-\zeta_{0}\right| \leqq 2 M C|\zeta-z|$. So with these assumptions,

$$
\begin{aligned}
\int_{\partial D \cap s_{\xi_{0}, \delta}}|f(\zeta)| \frac{|K(\zeta, z)|}{\left|\Phi^{n}(\zeta, z)\right|} d \sigma(\zeta) & \leqq\|f\|_{\infty} 2 M C \int_{\partial D \cap s_{\delta_{0}, \delta}} \frac{|\zeta-z|}{\left|\Phi^{n}(\zeta, z)\right|} d \sigma(\zeta) \\
& \leqq 2 M C\|f\|_{\infty} \int_{\partial D \cap s_{z_{2}, 2 \delta}} \frac{|\zeta-z|}{\left|\Phi^{n}(\zeta, z)\right|} d \sigma(\zeta) \\
& \leqq 2 M C\|f\|_{\infty} \gamma 2 \delta \log \frac{1}{2 \delta}, \text { if } 2 \delta<\delta_{0}
\end{aligned}
$$

So now if we first choose $\delta$ sufficiently small and then let $z$ approach $\zeta_{0}$ 
along the inward normal we see that 2.5 approaches zero. This proves 2.4. Now it is easy to finish the proof of the theorem. We take $f \in A(D)$ such that $f\left(\zeta_{0}\right)=1$ and $|f(\zeta)|<1$ for $\zeta \in \bar{D} \mid\left\{\zeta_{0}\right\}$. Applying 2.4 to $f^{N}$ we get

$$
1=f^{N}\left(\zeta_{0}\right)=\int_{\partial D} f^{N}(\zeta) \frac{K\left(\zeta, \zeta_{0}\right)}{\Phi^{n}\left(\zeta, \zeta_{0}\right)} d \sigma(\zeta)
$$

However the right hand side approaches zero, by the bounded convergence theorem. This contradiction completes the proof of Theorem A.

We now apply Theorem A to obtain

THEOREM B. Suppose D is a bounded strictly pseudoconvex domain in $\mathrm{C}^{n}$ with a $C^{3}$ boundary. There is a linear mapping $T: A(D) \rightarrow$ $H(D \times D)^{n}$ such that $(T f), \in C[(\bar{D} \times \bar{D}) \backslash\{(z, z): z \in \partial D\}]$ for every $f \in$ $A(D)$ and such that

$$
f(z)-f(\omega)=\sum\left(z_{\imath}-\omega_{i}\right)(T f)_{\imath}(z, \omega)
$$

Proof. From Henkin's integral formula we see that

$$
f(z)-f(\omega)=\int f(\zeta) \frac{\Phi^{n}(\zeta, \omega) K(\zeta, z)-\Phi^{n}(\zeta, z) K(\zeta, \omega)}{\Phi^{n}(\zeta, z) \Phi^{n}(\zeta, \omega)} d \sigma(\zeta)
$$

If $\quad L(\zeta, z, \omega)=\Phi^{n}(\zeta, \omega) K(\zeta, z)-\Phi^{n}(\zeta, z) K(\zeta, \omega)$, then $L \in C^{1}\left(V_{\epsilon}\right.$, $\left.H\left(D_{\epsilon} \times D_{\epsilon}\right)\right)$ and $L(\zeta, z, z) \equiv 0$, so by the argument given as a remark on page 148 of [8] there are functions $L_{t} \in C^{1}\left(V_{\epsilon^{\prime}}, H\left(D_{\epsilon^{\prime}} \times D_{\epsilon^{\prime}}\right)\right.$ ) (for some $\epsilon^{\prime}<\epsilon$ ) such that

$$
L(\zeta, z, \omega)=\sum_{i=1}^{n}\left(z_{i}-\omega_{\imath}\right) L_{i}(\zeta, z, \omega)
$$

Hence, we have

$$
f(z)-f(\omega)=\sum_{i=1}^{n}\left(z_{\imath}-\omega_{\imath}\right) \int f(\zeta) \frac{L_{i}(\zeta, z, \omega)}{\Phi^{n}(\zeta, z) \Phi^{n}(\zeta, \omega)} d \sigma(\zeta)
$$

So it remains to show that

$$
f_{i}(z, \omega)=\int f(\zeta) \frac{L_{i}(\zeta, z, \omega)}{\Phi^{n}(\zeta, z) \Phi^{n}(\zeta, \omega)} d \Phi(\zeta)
$$


satisfies the statement of the theorem. Certainly $f_{i} \in H(D \times D)$ so we need only show that $f_{\imath} \in C[(\bar{D} \times \bar{D}) \backslash\{(z, z): z \in \partial D\}]$. Suppose $(z, \omega) \in$ $D \times D$ and $(z, \omega) \rightarrow\left(\zeta_{0}, \omega_{0}\right) \in \bar{D} \times \bar{D} \backslash\{(z, z): z \in \partial D\}$. We wish to show that $f_{\imath}(z, \omega)$ has a limit. We will assume that $\zeta_{0} \in \partial D$ and $\omega_{0} \in \partial D$ and $\zeta_{0} \neq \omega_{0}$. The other possibilities are treated in a similar fashion (and are easier). By Theorem $\mathrm{A}, K\left(\zeta_{0}, \zeta_{0}\right) \neq 0$, and $K\left(\omega_{0}, \omega_{0}\right) \neq 0$. Hence there is a $\delta>0$ such that if $\left|z-\zeta_{0}\right| \leqq 2 \delta$ and $\left|\zeta-\zeta_{0}\right| \leqq 2 \delta$ then $K(z, \zeta) \neq 0$, and if $\left|z-\omega_{0}\right| \leqq 2 \delta$ and $\left|\zeta-\omega_{0}\right| \leqq 2 \delta$ then $K(z, \zeta) \neq 0$. We also assume $4 \delta<\left|\zeta_{0}-\omega_{0}\right|$. Let $\varphi(z)$ be a $C^{\infty}$ function that is identically equal to 1 if $|z| \leqq \delta^{2}$ and identically 0 if $|z| \geqq(2 \delta)^{2}$. Now we write

$$
\begin{aligned}
f_{\imath}(z, \omega)= & \int f(\zeta) \frac{L_{i}(\zeta, z, \omega) \varphi\left(|z-\zeta|^{2}\right)}{K(\zeta, z) \Phi^{n}(\zeta, \omega)} \frac{K(\zeta, z)}{\Phi^{n}(\zeta, z)} d \sigma(\zeta) \\
& +\int f(\zeta) \frac{L_{i}(\zeta, z, \omega) \varphi\left(|\omega-\zeta|^{2}\right)}{K(\zeta, \omega) \Phi^{n}(\zeta, z)} \frac{K(\zeta, \omega)}{\Phi^{n}(\zeta, \omega)} d \sigma(\zeta) \\
& +\int f(\zeta) \frac{L_{t}(\zeta, z, \omega)}{\Phi^{n}(\zeta, z) \Phi^{n}(\zeta, \omega)}\left[1-\varphi\left(|z-\zeta|^{2}\right)-\varphi\left(|\omega-\zeta|^{2}\right)\right] d \sigma(\zeta)
\end{aligned}
$$

for $\left|z-\zeta_{0}\right|<\delta$ and $\left|\omega-\omega_{0}\right|<\delta$. The third term has a limit as $(z, \omega) \rightarrow\left(\zeta_{0}, \omega_{0}\right)$ since we may take the limit under the integral sign. We write the first term as

2.6.

$$
\int f(\zeta) \chi(\zeta, z, \omega) \frac{K(\zeta, z)}{\Phi^{n}(\zeta, z)} d \sigma(\zeta)
$$

where all we need to know about $\chi$ is that it is continuous on $\partial D \times \bar{D} \times S_{\omega 0, \delta}$ and that there is a constant $C$ such that $\mid \chi(\zeta, z, \omega)-$ $\chi\left(\zeta^{\prime}, z, \omega\right) \leqq C\left|\zeta-\zeta^{\prime}\right|$, for all $z, \omega, \zeta$, and $\zeta^{\prime}$. Now we just imitate the proof of Lemma 4.3 of [3] to see that 2.6 has a limit as $(z, \omega) \rightarrow\left(\zeta_{0}, \omega_{0}\right)$. The second term is handled in the same way as the first. This completes the proof.

Note that if

$$
f(z)-f(\omega)=\sum_{\imath=1}^{n}\left(z_{\imath}-\omega_{\imath}\right) g_{\imath}(z, \omega) \text { then } \frac{\partial f}{\partial z_{\imath}}(z)=g_{\imath}(z, z)
$$

so so that $g_{i}$ need not be in $A(D \times D)$ when $f \in A(D)$.

\section{REFERENCES}

1. S. Bergman, Über die Kernfunktion eines Bereiches und ihr Verhalten am Rande, I-II., J. Reine Angew. Math., 169 (1933), 1-42, and 172 (1935), 89-128.

2. A. Gleason, Finitely generated ideals in Banach algebras, J. Math. Mech., 13 (1964), 125-132. 
3. G. Henkin, Integral representations of functions holomorphic in strictly pseudoconvex domains and some applications, Mat. Sb., 78 (120) (1969), 597-616.

4. - The approximation of functions in pseudoconvex domains and a Theorem of $Z, L$. Leibenzan. Bull. Acad. Polon. Sci. Ser. Sci. Math. Astron. Phys., 19 (1971), 37-42.

5. L. Hörmander, $L^{2}$ estimates and existence theorems for the $\bar{\partial}$ operator, Acta Math., 113 (1965), $89-152$.

6. N. Kerzman and A. Nagel, Finitely generated ideals in certain function algebras, J. Functional Analysis, 7 (1971), 212-215.

7. I. Lieb, Die Cauchy-Riemannischen Differential-gleichungen auf streng pseudokonvexen Gebieten, Beschrankte Lösungen, Math. Ann., 190 (1970), 6-44.

8. N. Øvrelid, Integral representation formulas and $L^{p}$ estimates for the $\bar{\partial}$ equation, Math. Scand., 29 (1971), 137-160.

9. —_ Generators of the maximal ideals of $A(\bar{D})$, Pacific J. Math., 39, no. 1, (1971), 219-223.

Received March 11, 1976. The first author was partially supported by an NSF grant. The second author was partially supported by a grant from the research foundation of CUNY.

UNIVERSITY OF WISCONSIN-MADISON

AND

Herbert Lehman Colíege of CUNy 



\section{Pacific Journal of Mathematics}

\section{Vol. 66, No. $1 \quad$ November, 1976}

Helen Elizabeth. Adams, Factorization-prime ideals in integral domains ............ Patrick Robert Ahern and Robert Bruce Schneider, The boundary behavior of Henkin's kernel.

Daniel D. Anderson, Jacob R. Matijevic and Warren Douglas Nichols, The Krull

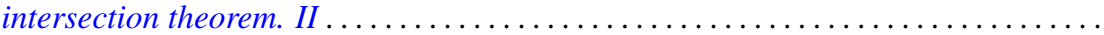

Efraim Pacillas Armendariz, On semiprime P.I.-algebras over commutative regular

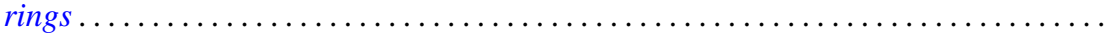

Robert H. Bird and Charles John Parry, Integral bases for bicyclic biquadratic fields

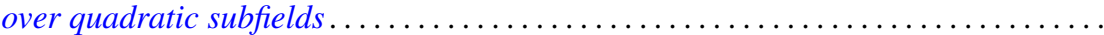

Tae Ho Choe and Young Hee Hong, Extensions of completely regular ordered

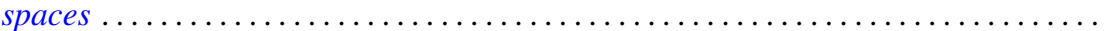

John Dauns, Generalized monoform and quasi injective modules ...............

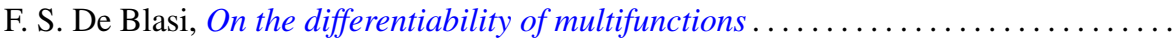

Paul M. Eakin, Jr. and Avinash Madhav Sathaye, R-endomorphisms of $R[[X]]$ are

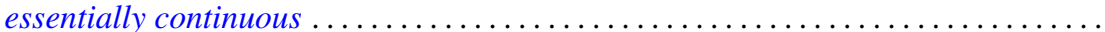

Larry Quin Eifler, Open mapping theorems for probability measures on metric

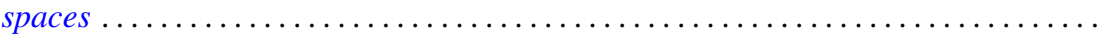

Garret J. Etgen and James Pawlowski, Oscillation criteria for second order self adjoint

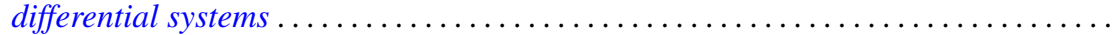

Ronald Fintushel, Local $S^{1}$ actions on 3-manifolds .

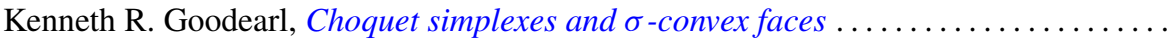

John R. Graef, Some nonoscillation criteria for higher order nonlinear differential

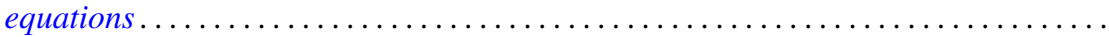

Charles Henry Heiberg, Norms of powers of absolutely convergent Fourier series: an example.

Les Andrew Karlovitz, Existence of fixed points of nonexpansive mappings in a space

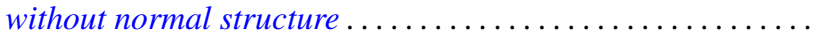

Gangaram S. Ladde, Systems of functional differential inequalities and functional

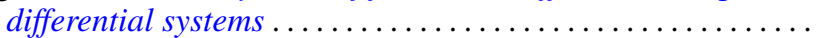

Joseph Michael Lambert, Conditions for simultaneous approximation and interpolation with norm preservation in $C[a, b]$.

Ernest Paul Lane, Insertion of a continuous function.

Robert F. Lax, Weierstrass points of products of Riemann surfaces .

Dan McCord, An estimate of the Nielsen number and an example concerning the Lefschetz fixed point theorem...

Paul Milnes and John Sydney Pym, Counterexample in the theory of continuous functions on topological groups...

Peter Johanna I. M. De Paepe, Homomorphism spaces of algebras of holomorphic functions

Judith Ann Palagallo, A representation of additive functionals on $L^{p}$-spaces,

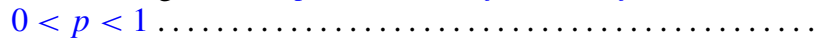

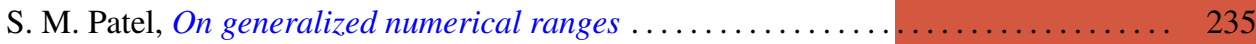

Thomas Thornton Read, A limit-point criterion for expressions with oscillatory

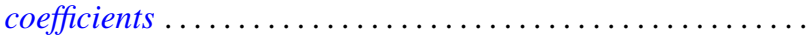

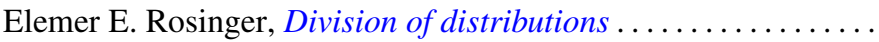

Peter S. Shoenfeld, Highly proximal and generalized almost finite

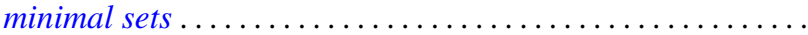

R. Sirois-Dumais and Stephen Willard, Quotient-universal sequential spaces

Robert Charles Thompson, Convex and concave functions of singular values of matrix sums....

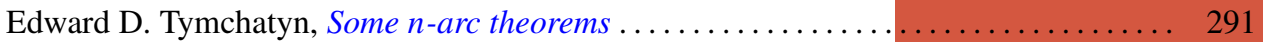

